Alma Mater Studiorum - Università di Bologna DEPARTMENT OF ECONOMICS

\title{
Investigating the Strategic Nature of Supply Functions in Oligopoly
}

Flavio Delbono

Luca Lambertini

Quaderni - Working Paper DSE $N^{\circ} 1011$

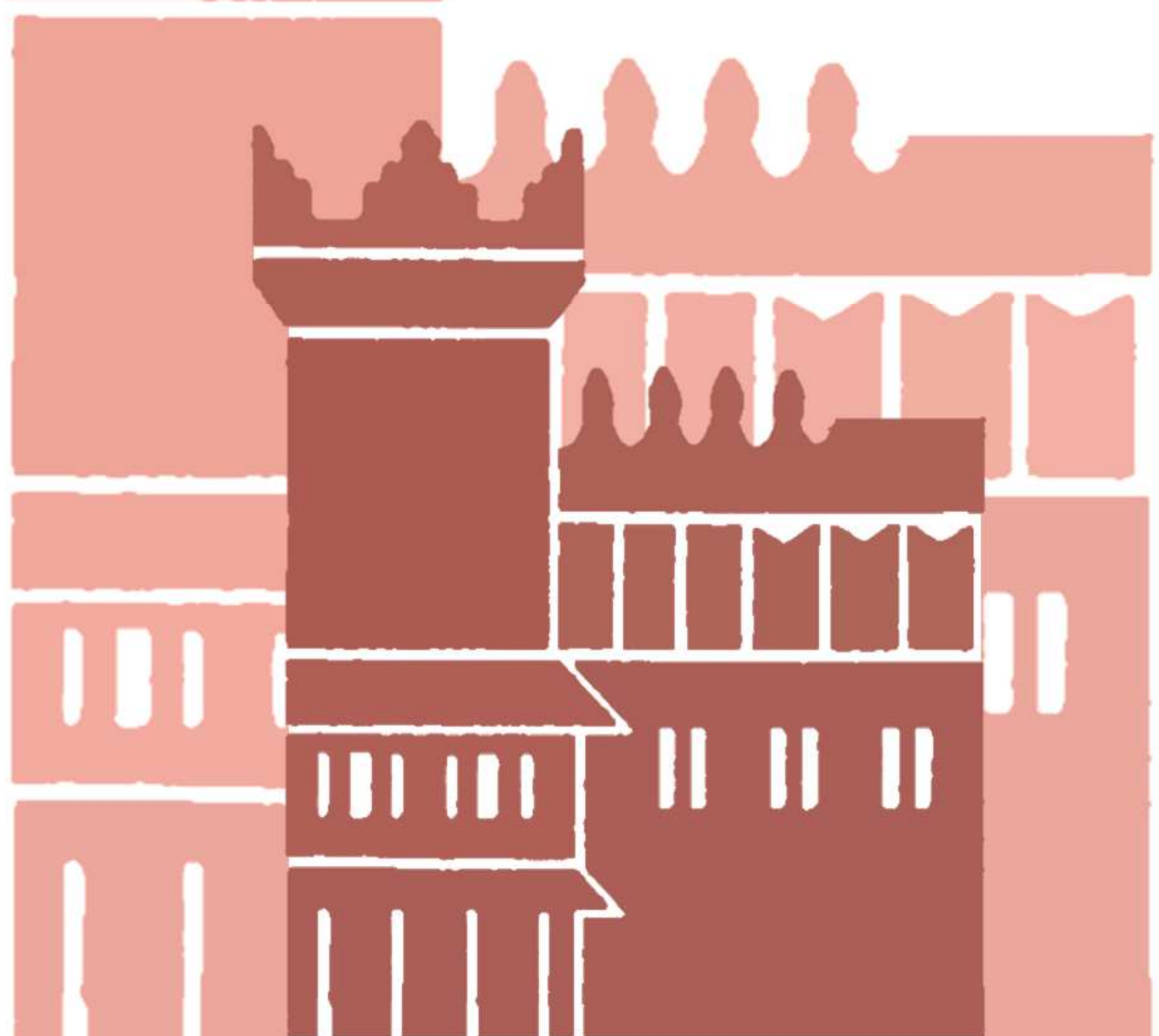




\title{
Investigating the Strategic Nature of Supply Functions in Oligopoly
}

\author{
Flavio Delbono ${ }^{\#}$ and Luca Lambertini ${ }^{\S}$ \\ \# Department of Economics, University of Bologna \\ Piazza Scaravilli 2, 40126 Bologna, Italy \\ flavio.delbono@unibo.it \\ $\S$ Department of Economics, University of Bologna \\ Strada Maggiore 45, 40125 Bologna, Italy \\ luca.lambertini@unibo.it
}

June 9, 2015 


\begin{abstract}
We show that supply functions cannot be classified as either strategic complements or substitutes according to the twofold criterion advanced by Bulow et al. (1985). This is because while the slope of the best reply is univocally positive, this is not the case with the sign of the cross derivative of marginal profit. We first show this discrepancy in the original Klemperer and Meyer (1989) setting, and then in a linear-quadratic model of differentiated duopoly. We further confirm and strengthen our result by proving that the game in supply functions is neither supermodular nor submodular.
\end{abstract}

JEL Codes: D43, L13

Keywords: supply function; lattice theory; strategic complements; strategic substitutes; super/submodularity 


\section{Introduction}

In a widely cited paper, Bulow et al. (1985) introduce an important criterion to classify the strategic nature of different choice variables in oligopolistic games. Borrowing a well established terminology from demand theory, they identify a binary taxonomy opposing strategic complements to strategic substitutes. Focusing on price or quantity competition in a single market, it turns out that one may detect the strategic nature of a choice variable by looking at the sign of the first derivative of the reaction function (the slope) or at the sign of the second mixed derivative of the payoff function, for both derivatives share the same sign under fairly general regularity conditions. If such a sign is positive, then the choice variable is said to be a strategic complement (e.g., price); if it is negative, the choice variable is said to be a strategic substitute (e.g., output). This taxonomy belongs by now to the advanced Industrial Organization jargon (see, for instance, Tirole, 1988 or Vives, 1999).

The main question addressed in this paper deals with the classification of supply functions. Competition in supply functions represent an interesting alternative to price and/or quantity competition when modelling oligopolistic market games. After the pioneering contributions by Grossman (1981) and especially Klemperer and Meyer (1989), the literature on supply functions has been growing, also because of the widespread opinion that supply function competition fits better than price or quantity competition our understanding of some key sectors (e.g., wholesale electricity). ${ }^{1}$

We approach our central question first in the original Klempeer and Meyer (1989) setting, and then within a model of (exogenously) differentiated duopoly where we concentrate on linear supply functions. When performing the test needed to classify the strategic nature of supply functions,

\footnotetext{
${ }^{1}$ See, for instance, Delgado and Moreno (2004), Ciarreta and Gutierrez-Hita (2006), Vives (2011), Holmberg et al. (2013), Delbono and Lambertini (2015a,b). For an interesting sample of the huge applied literature, see Bolle (1992) and Green and Newbery (1982).
} 
we show that while the sign of the reaction function slope is unambiguously positive, the sign of the second mixed derivative of the profit function may not be taken for granted. Except along the best reply, the latter sign may indeed be both positive and negative. Hence, as opposed to what happens with both price and quantity competition, under supply function competition there seem to be a discrepancy between the two (apparently equivalent) tests involved in the above classification. This discrepancy poses an obvious problem. Only after a detailed investigation of the second mixed derivative of the profit function, we succeed to establish that in the admissible range of the parameters its sign is positive too. However, we may not conclude that supply functions are strategic complements because the variation of marginal profits has not the same sign over the whole strategy space. This is confirmed by the lattice theory approach (Tarski, 1955; Topkis, 1978; 1979), whereby we show that the ultimate explanation of the general discrepancy between the two signs lies in the supply function game being not supermodular. ${ }^{2}$

The paper is organized as follows. In the next section we use the Klemperer and Meyer (1989) model and we perform the analysis needed to test the strategic nature of supply functions along the lines of Bulow et al. (1985). In section 3 we set up a linear-quadratic version of the Singh and Vives (1984) model which can be analytically solved in linear supply functions, as illustrated in section 4 . In section 5, we use the tools of lattice theory to generalize and motivate our findings. Section 6 concludes.

\section{Preliminaries}

We start from the original model of Klemperer and Meyer (1989). They assume that the industry demand curve is $Q=D(p)$, where $D(p)$ is twice continuously differentiable, strictly decreasing and concave in price $p$, over the relevant price range wherein $D(p) \geq 0$. The industry is a duopoly in

\footnotetext{
${ }^{2}$ For more on supermodular games, see Vives (1990), Milgrom and Roberts (1990) and Milgrom and Shannon (1994), inter alia.
} 
which firms have identical cost functions $C\left(q_{i}\right)$, with $C^{\prime}\left(q_{i}\right), C^{\prime \prime}\left(q_{i}\right) \geq 0$ for all $q_{i} \geq 0$. Each firms chooses its supply function $S_{i}(p)$ under the market clearing condition $D\left(p^{*}\right)=S_{i}\left(p^{*}\right)+S_{j}\left(p^{*}\right)$, assuming unicity of the marketclearing price $p^{*}$. Firm $i$ 's profit function is therefore defined as $\pi_{i}(p)=$ $p S_{i}(p)-C\left(S_{i}(p)\right)$, which, under market clearing, writes as follows:

$$
\pi_{i}(p)=p\left[D(p)-S_{j}(p)\right]-C\left(D(p)-S_{j}(p)\right)
$$

Firm $i$ 's problem consists in maximising (1) w.r.t. $p$, which implies solving the following first order condition (FOC):

$$
\frac{\partial \pi_{i}(p)}{\partial p}=D(p)-S_{j}(p)+\left[p-C^{\prime}\left(D(p)-S_{j}(p)\right)\right]\left[D^{\prime}(p)-S_{j}^{\prime}(p)\right]=0
$$

to obtain

$$
S_{j}^{\prime}(\bar{p})=\frac{\bar{q}_{i}}{\bar{p}-C^{\prime}\left(\bar{q}_{i}\right)}+D^{\prime}(\bar{p})
$$

where $S_{j}(\bar{p})=\bar{q}_{j}$ and consequently $\bar{q}_{i}=D(\bar{p})-S_{j}(\bar{p})$. As in Klemperer and Meyer (1989, p. 1248), we shall confine our attention to the case in which $S_{j}^{\prime}(\bar{p}) \geq 0$, i.e., supply functions are required to have non-negative slopes at $p=\bar{p}$, where $\bar{p}$ is a market-clearing price granting a positive mark-up.

We now try to detect whether supply functions are strategic complements or substitutes by using the twofold test introduced by Bulow et al. (1985). Accordingly, we must (i) identify the best reply function of firm $i$ in the space of supply functions and check the sign of its slope w.r.t. the rival's; (ii) determine the sign of the partial derivative of firm $i$ 's marginal profit w.r.t. the rival's supply function; and (iii) compare the two signs. If the argument put forward by Bulow et al. (1985) in the space of either prices or quantities holds true also in the space of supply functions, the two signs should coincide, allowing one to establish the strategic nature of supply functions.

As a first step, we pose $D(p)-S_{j}(p)=S_{i}(p)$ in $(2)$ and then solve it w.r.t. $S_{i}(p)$ to obtain the best reply of firm $i$ to the rival's supply function:

$$
S_{i}^{*}(\bar{p})=-\left(\bar{p}-C^{\prime}\left(D(\bar{p})-S_{j}(\bar{p})\right)\right)\left(D^{\prime}(\bar{p})-S_{j}^{\prime}(\bar{p})\right)
$$


The partial derivative of $S_{i}^{*}(\bar{p})$ w.r.t. $S_{j}(\bar{p})$ is

$$
\frac{\partial S_{i}^{*}(\bar{p})}{\partial S_{j}(\bar{p})}=-\left(D^{\prime}(\bar{p})-S_{j}^{\prime}(\bar{p})\right) C^{\prime}\left(D(\bar{p})-S_{j}(\bar{p})\right) \geq 0
$$

because $D^{\prime}(\bar{p})-S_{j}^{\prime}(\bar{p})<0$ as $D^{\prime}(\bar{p})<0$ and $S_{j}^{\prime}(\bar{p}) \geq 0$. Hence, best replies in the supply function space are non-decreasing.

The second step amounts to evaluating the sign of the second cross derivative

$$
\frac{\partial^{2} \pi_{i}(p)}{\partial S_{i}(p) \partial S_{j}(p)}
$$

measuring the effect of a change in $S_{j}(p)$ on firm $i$ 's marginal profit $\partial \pi_{i}(p) / \partial S_{i}(p)$. The latter obtains by observing that

$$
\frac{\partial \pi_{i}(p)}{\partial p}=\frac{\partial \pi_{i}(p)}{\partial S_{i}(p)} \cdot \frac{\partial S_{i}(p)}{\partial p}
$$

which rewrites as

$$
\frac{\partial \pi_{i}(p)}{\partial p}=\frac{\partial \pi_{i}(p)}{\partial S_{i}(p)} \cdot S_{i}^{\prime}(p)
$$

where $S_{i}^{\prime}(p)=\partial S_{i}(p) / \partial p$. Consequently, provided $S_{i}(p)$ is invertible,

$$
\begin{gathered}
\frac{\partial \pi_{i}(p)}{\partial S_{i}(p)}=\frac{\partial \pi_{i}(p)}{\partial p} \cdot \frac{\partial p}{\partial S_{i}}=\frac{\partial \pi_{i}(p)}{\partial p} \cdot \frac{1}{S_{i}^{\prime}(p)}= \\
\frac{D(p)-S_{j}(p)+\left[p-C^{\prime}\left(D(p)-S_{j}(p)\right)\right]\left[D^{\prime}(p)-S_{j}^{\prime}(p)\right]}{D^{\prime}(p)-S_{j}^{\prime}(p)}
\end{gathered}
$$

for any market-clearing price. Differentiating $(9)$ w.r.t. $S_{j}(p)$, we get:

$$
\frac{\partial^{2} \pi_{i}(p)}{\partial S_{i}(p) \partial S_{j}(p)}=\frac{-1+\left(D^{\prime}(p)-S_{j}^{\prime}(p)\right) C^{\prime}\left(D(p)-S_{j}(p)\right)}{D^{\prime}(p)-S_{j}^{\prime}(p)}
$$

The r.h.s. of $(10)$ is strictly positive for $p=\bar{p}$ as $S_{j}^{\prime}(\bar{p}) \geq 0$, for the same reasons whereby (5) is non-negative. However, since here we are considering any market clearing price, there may exist price levels $p \neq \bar{p}$ at which $S_{j}^{\prime}(p)<$ 0 and therefore (10) may change sign.

The above analysis boils down to the following conclusion. Unlike what happens in the space of prices or quantities, here the two tests suggested 
by Bulow et al. (1985) do not provide systematically the same answer for any price level ensuring market-clearing. Hence, we cannot conclude, in general, whether supply functions are strategic complements or substitutes. To disentangle this issue, we turn to a linear quadratic model.

\section{The linear-quadratic model}

As a workhorse, we consider the model of differentiated duopoly introduced by Singh and Vives (1984). The utility function of the representative consumer is

$$
U=a\left(q_{1}+q_{2}\right)-\frac{1}{2}\left(q_{1}^{2}+q_{2}^{2}+2 \sigma q_{1} q_{2}\right)
$$

where $q_{i}$ is the quantity of the variety supplied by firm $i=1,2, a>0$ and parameter $\sigma \in(0,1]$ measures the degree of product substitutability, i.e., $\sigma$ is an inverse measure of product differentiation. When $\sigma=1$, the product is homogeneous. ${ }^{3}$ The direct demand functions resulting from the constrained maximisation problem are:

$$
q_{i}=\max \left\{0, \frac{a}{1+\sigma}-\frac{p_{i}}{1-\sigma^{2}}+\frac{\sigma p_{j}}{1-\sigma^{2}}\right\}, i=1,2 .
$$

System (12) can be inverted to yield the direct demand system:

$$
p_{i}=a-q_{i}-\sigma q_{j}, i=1,2
$$

On the supply side, both single-product firms operate with the convex cost function $C_{i}=c q_{i}^{2} / 2$, with $c>0$. The profit function of firm $i$ is

$$
\pi_{i}=p_{i} q_{i}-\frac{c q_{i}^{2}}{2}
$$

To model competition in supply functions, for the sake of simplicity, we focus on linear supply functions. To this end, we follow the procedure illustrated in Ciarreta and Gutierrez-Hita (2006). The supply function of firm $i$

\footnotetext{
${ }^{3}$ If $\sigma=0$, the two varieties do not interact and firms are separate monopolists. We also disregard the range $\sigma \in[-1,0)$, where products are complements.
} 
is defined as $S_{i}=\beta_{i} p_{i}$, and the ex ante market clearing condition is $S_{i}=q_{i}$, where $q_{i}$ is defined as in (12). The presence of two varieties requires imposing two market-clearing conditions. Taking the individual demand function in (12), whenever $q_{i}>0$, market clearing requires imposing $\beta_{i} p_{i}=q_{i}$ :

$$
\beta_{i} p_{i}=\frac{a}{1+\sigma}-\frac{p_{i}}{1-\sigma^{2}}+\frac{\sigma p_{j}}{1-\sigma^{2}}
$$

Solving system (15) w.r.t. $p_{i}$ and $p_{j}$, the resulting market-clearing prices are:

$$
p_{i}=\frac{a\left[1+(1-\sigma) \beta_{j}\right]}{1+\beta_{j}+\left[1+\beta_{j}\left(1-\sigma^{2}\right)\right] \beta_{i}}
$$

The profit function of firm $i$ therefore writes as

$$
\pi_{i}=p_{i} S_{i}-\frac{c S_{i}^{2}}{2}=\beta_{i} p_{i}^{2}-\frac{c \beta_{i}^{2} p_{i}^{2}}{2}
$$

where $p_{i}$ is $(16)$.

Competition in supply function takes place in the space $\left(\beta_{1}, \beta_{2}\right)$, with $\beta_{1}, \beta_{2}>0$. The FOC for the maximisation of (17) w.r.t. $\beta_{i}$ is:

$$
\frac{\partial \pi_{i}}{\partial \beta_{i}}=\frac{a^{2}\left[1+(1-\sigma) \beta_{j}\right]^{2}\left[1+\beta_{j}-\left(1+c+\left(1+c-\sigma^{2}\right) \beta_{j}\right) \beta_{i}\right]}{\left[1+\beta_{j}+\left(1+\left(1-\sigma^{2}\right) \beta_{j}\right) \beta_{i}\right]^{3}}=0
$$

which generates the following best reply function: ${ }^{4}$

$$
\beta_{i}^{*}\left(\beta_{j}\right)=\frac{1+\beta_{j}}{1+c+\left(1+c-\sigma^{2}\right) \beta_{j}}
$$

\section{Testing linear supply functions}

We now investigate the strategic nature of supply functions. From (19), one easily verifies that the slope of the reaction function is

$$
\frac{\partial \beta_{i}^{*}\left(\beta_{j}\right)}{\partial \beta_{j}}=\frac{\sigma^{2}}{\left[1+c+\left(1+c-\sigma^{2}\right) \beta_{j}\right]^{2}}
$$

\footnotetext{
${ }^{4}$ The second order condition, which is omitted for brevity, is satisfied in correspondence of $\beta_{i}=\beta_{i}^{*}\left(\beta_{j}\right)$.
} 
which is clearly positive everywhere. Therefore, best replies are increasing, which, in the jargon dating back to Bulow et al. (1985), corresponds to the case of strategic complementarity. However, in the present setting, this derivative is

$$
\frac{\partial^{2} \pi_{i}}{\partial \beta_{i} \partial \beta_{j}}=\frac{a^{2} \sigma\left[1-\left(1-\sigma^{2}\right) \beta_{j}\right] \Theta}{\left[1+\beta_{j}+\left(1+\left(1-\sigma^{2}\right) \beta_{j}\right) \beta_{i}\right]^{4}}
$$

where

$$
\begin{gathered}
\Theta \equiv \beta_{i}^{2}\left[2(1-\sigma)-c(3 \sigma-2)+(1-\sigma)\left[2\left(1-\sigma^{2}\right)+c(2-\sigma)\right] \beta_{j}\right]+ \\
\beta_{i}\left[4 \sigma\left(1+\beta_{j}(1-\sigma)\right)+2 c\left(1+\beta_{j}\right)\right]-2\left(1+\beta_{j}\right)
\end{gathered}
$$

To ease the exposition, define

$$
\Upsilon \equiv 2(1-\sigma)-c(3 \sigma-2)+(1-\sigma)\left[2\left(1-\sigma^{2}\right)+c(2-\sigma)\right] \beta_{j}
$$

Then clearly,

- $\partial^{2} \pi_{i} / \partial \beta_{i} \partial \beta_{j}$ is convex (resp., concave) in $\beta_{i}$ if $\Upsilon>0$ (resp., $\Upsilon<0$ ) and

- the sign of (21) is the sign of $\Theta$, which is equal to zero in correspondence of

$$
\beta_{i}=-\frac{c+2 \sigma+(c+2 \sigma(1-\sigma)) \beta_{j} \pm \sqrt{\Psi}}{2(1-\sigma)-c(3 \sigma-2)+(1-\sigma)\left[2\left(1-\sigma^{2}\right)+c(2-\sigma)\right] \beta_{j}}
$$

where

$$
\begin{gathered}
\Psi \equiv 2\left(1+\beta_{j}\right)\left[\left(2+\left(2\left(1-\sigma^{2}\right)+c(2-\sigma)\right) \beta_{j}\right)(1-\sigma)+c(2-3 \sigma)\right]+ \\
{\left[c\left(1+\beta_{j}\right)+2 \sigma\left(1+(1-\sigma) \beta_{j}\right)\right]^{2}}
\end{gathered}
$$

Now note that

$$
\beta_{i+}-\beta_{i-}=-\frac{\sqrt{\Psi}}{\Upsilon}
$$

where the sign of the denominator may change precisely when $\partial^{2} \pi_{i} / \partial \beta_{i} \partial \beta_{j}$ changes from convex to concave. As it is shown in appendix, $\Psi>0$, so that $\beta_{i+} \neq \beta_{i-}$ and $\beta_{i+}, \beta_{i-} \in \mathbb{R}$. Looking at $\Upsilon$, it appears that 
Lemma $1 \sigma \in(0,2 / 3]$ suffices to ensure $\Upsilon>0$; therefore, in the same range, $\beta_{i-}>\beta_{i+}$ and $\partial^{2} \pi_{i} / \partial \beta_{i} \partial \beta_{j}>0$ outside the interval $\left[\beta_{i+}, \beta_{i-}\right]$. Instead, $\sigma \in(2 / 3,1]$ is necessary but not sufficient to yield $\beta_{i+}>\beta_{i-}$.

Let us now focus on the range $\sigma \in(2 / 3,1]$. Here, in the denominator of (24), $2(1-\sigma)-c(3 \sigma-2)<0$ for all

$$
c>\frac{2(1-\sigma)}{3 \sigma-2} \equiv \widehat{c} \geq 0 \forall \sigma \in(2 / 3,1]
$$

Then, for all $c>\widehat{c}, \Upsilon \gtreqless 0$ for all

$$
\beta_{j} \gtreqless \frac{c(3 \sigma-2)-2(1-\sigma)}{(1-\sigma)\left[2\left(1-\sigma^{2}\right)+c(2-\sigma)\right]} \equiv \widehat{\beta}_{j}>0
$$

and

$$
\widehat{\beta}_{j}>\widehat{c} \forall c>\frac{2(1-3 \sigma)+4 \sigma^{2}(2-\sigma)}{\sigma(1+2 \sigma)-2} \equiv \widetilde{c} \geq 0 \forall \sigma \in(2 / 3,1]
$$

Moreover, $\widetilde{c} \geq \widehat{c} \forall \sigma \in(2 / 3,1]$. This produces the graph in Figure 1, where $\widetilde{c}$ and $\widehat{c}$ are drawn. The signs appearing in the four relevant regions are the sign of $\partial^{2} \pi_{i} / \partial \beta_{i} \partial \beta_{j}$. 
Figure 1 The sign of $\partial^{2} \pi_{i} / \partial \beta_{i} \partial \beta_{j}$

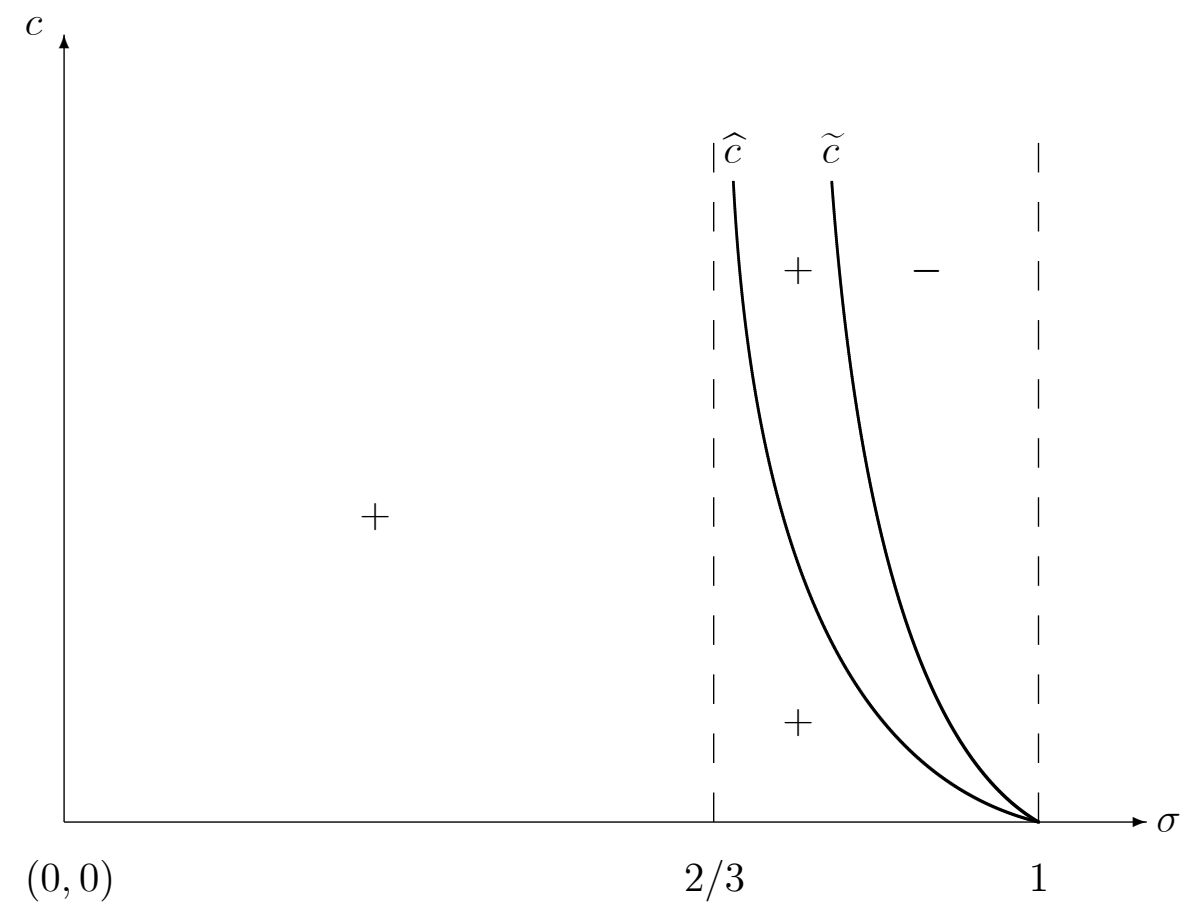

The foregoing analysis boils down to the following

Proposition 2 For all $\sigma \in(2 / 3,1]$ and $c>\widetilde{c}, \partial^{2} \pi_{i} / \partial \beta_{i} \partial \beta_{j}<0$, while $\partial^{2} \pi_{i} / \partial \beta_{i} \partial \beta_{j}>0$ anywhere else.

This result illustrates that, while in a standard duopoly model where under Bertrand or Cournot competition the second cross derivative of profits has the same sign everywhere, under supply function competition this does not apply in general. Moreover, again under Bertrand or Cournot behaviour, the sign of the second cross derivative of profits coincides with the sign of the slope of the best reply function. This, in general, does not hold under supply function competition. However, substituting the best reply (19) into 
(21), or equivalently into (22), one obtains:

$$
\left.\Theta\right|_{\beta_{i}=\beta_{i}^{*}\left(\beta_{j}\right)}=\frac{\sigma\left(1+\beta_{j}\right)\left[1+(1-\sigma) \beta_{j}\right]\left[2+c+\left(2\left(1-\sigma^{2}\right)+c\right) \beta_{j}\right]}{\left[1+c+\left(1+c-\sigma^{2}\right) \beta_{j}\right]^{2}}>0
$$

everywhere. Hence, along the best reply the sign of (20) and (21) is indeed the same. To explain this result, without further ado, one can easily locate $\beta_{i}^{*}\left(\beta_{j}\right)$ w.r.t. $\beta_{i+}$ and $\beta_{i-}$. It turns out that:

- if $\Upsilon>0$, then $\beta_{i}^{*}\left(\beta_{j}\right)>\beta_{i-}>\beta_{i+}$;

- if $\Upsilon<0$, then $\beta_{i}^{*}\left(\beta_{j}\right) \in\left(\max \left\{0, \beta_{i-}\right\}, \beta_{i+}\right)$.

These alternative scenarios are represented in Figure 2a,b.

Figure 2a The case $\Upsilon>0$

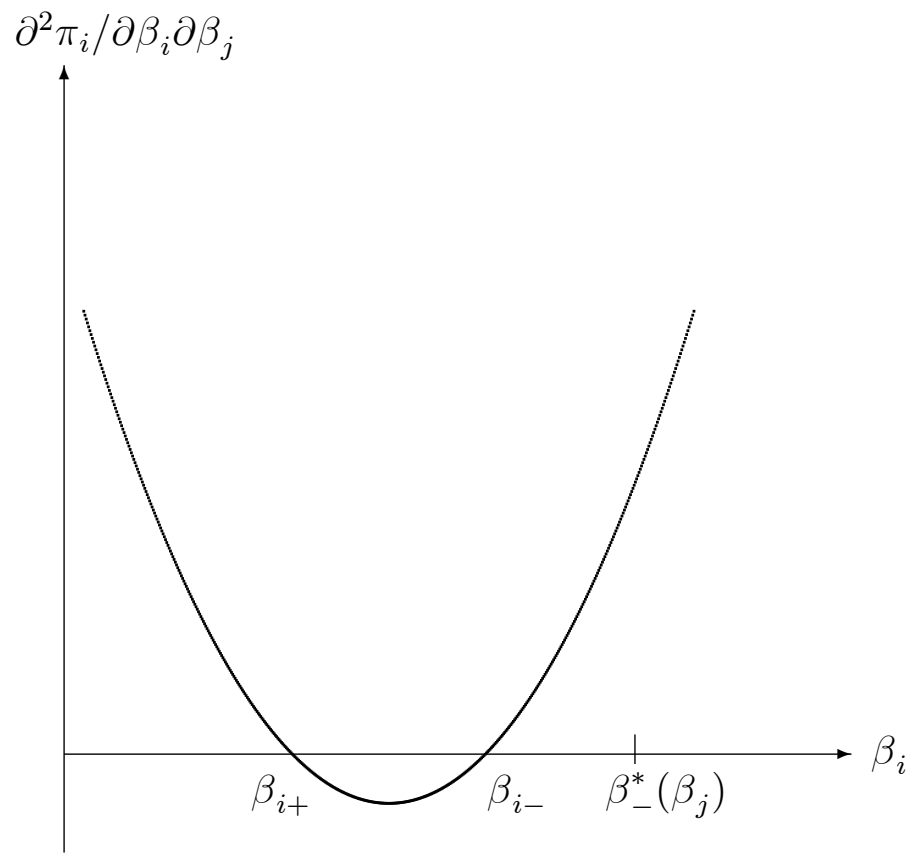


Figure $\mathbf{2 b}$ The case $\Upsilon<0$

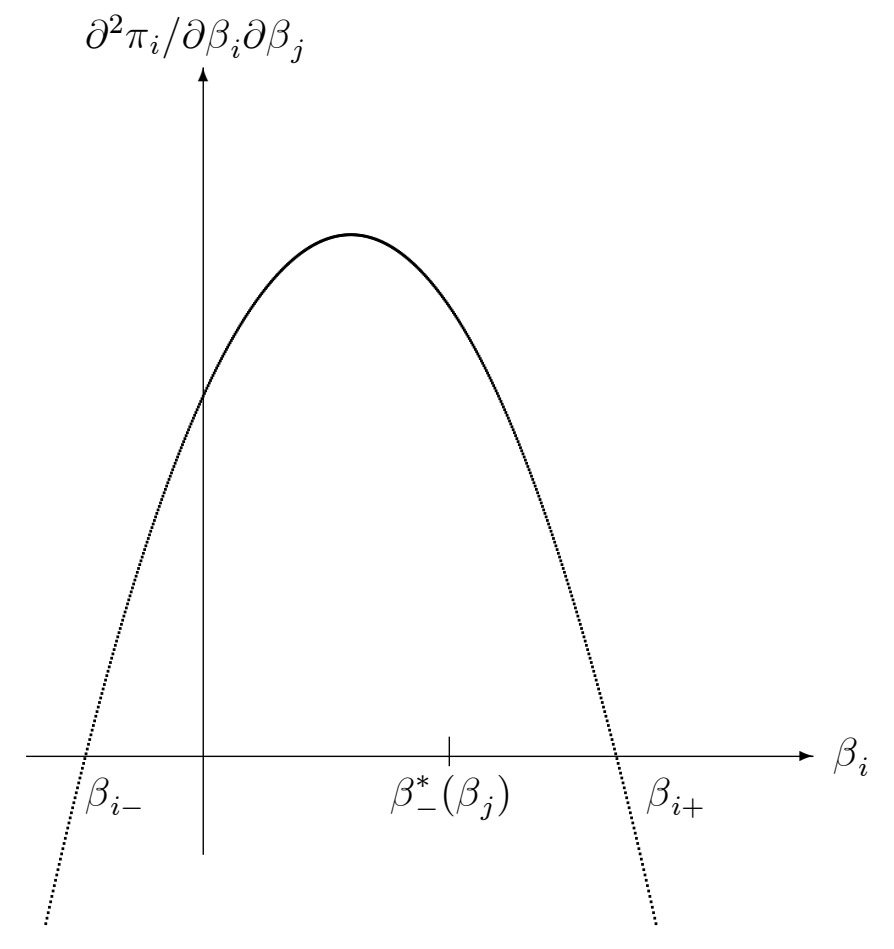

So far, we have proved that the equivalence between the two criteria suggested by Bulow et al. (1985) to detect whether strategic variables are complements or substitutes stop holding true under supply function competition. Indeed, while the sign of the slope of the best reply is univocally positive (suggesting the presence of strategic complementarity), the sign of the second cross derivative of profits is not (although it is positive along the best reply). This discrepancy between the two signs, in particular the fact that the effect of a change in a player's strategy on the opponent's marginal profits has not the same sign everywhere, prevents one from classifying the present game as one in which either strategic complementarity or strategic substitutability prevails. 
The acquired wisdom (see, e.g., Vives, 1999) holds that if the two signs are both positive (negative) everywhere, so that strategic variables are complements (substitutes), then the game is supermodular (submodular) in the jargon of lattice theory. Since, in the present setting, the two signs do not coincide everywhere, the direct implication stemming from the above argument is that the game played in the space of supply functions may not be classified as either supermodular or submodular. This analysis is carried out in the next section.

\section{The lattice approach to supply function com- petition}

The property of strategic complementarity is captured by both supermodularity and increasing differences. The latter concept is defined as follows. Let $X$ be a lattice and $\Xi$ a partially ordered set. The function $\pi_{i}\left(x_{i}, x_{j}\right)$ : $X \times \Xi \rightarrow \mathbb{R}$ has (strictly) increasing differences in its arguments $x_{i}$ and $x_{j}$ if $\pi_{i}\left(x_{i}, x_{j}\right)-\pi_{i}\left(x_{i}, x_{j}^{\prime}\right)$ is (strictly) increasing in $x_{i}$ for all $x_{j} \geq x_{j}^{\prime}$. By replacing 'increasing' with 'decreasing' one obtains the definition of decreasing differences.

The concept of supermodularity is stronger than that of increasing differences. However, if the function $\pi\left(x_{i}, x_{j}\right)$ is defined over a product of ordered sets, the two coincide. Moreover, if $\pi_{i}\left(x_{i}, x_{j}\right)$ is twice continuously differentiable over the space $X$, then $\pi\left(x_{i}, x_{j}\right)$ is supermodular iff $\partial \pi\left(x_{i}, x_{j}\right) / \partial x_{i} \partial x_{j} \geq 0$ for all $x_{i}, x_{j} \in X, j \neq i$ (see Vives, 1999, pp. 2425). The latter is nothing but one of the two conditions by which Bulow et al. (1985) identify equivalently strategic complementarity, the other one being increasing best replies.

In lattice theory, the function $\pi\left(x_{i}, x_{j}\right)$ is supermodular (submodular) iff (see Amir, 1996, p. 135, inequality (1.1)):

$$
\pi\left(x_{i A}, x_{j A}\right)-\pi\left(x_{i B}, x_{j A}\right) \geq(\leq) \pi\left(x_{i A}, x_{j B}\right)-\pi\left(x_{i B}, x_{j B}\right)
$$


for all $x_{i A} \geq x_{i B}$ and $x_{j A} \geq x_{j B}$.

Now we pose $x_{i}=\beta_{i}$ and $x_{j}=\beta_{j} ; \beta_{i A}=\beta_{j A}=\beta_{A} ; \beta_{i B}=\beta_{j B}=\beta_{B}$, with $\beta_{A} \geq \beta_{B}$. Then, we use the profit function (17). Accordingly, dropping the index as it is redundant, we have

$$
\begin{gathered}
\pi\left(\beta_{A}, \beta_{A}\right)=\frac{a^{2} \beta_{A}\left(2-c \beta_{A}\right)}{2\left[1+\beta_{A}(1+\sigma)\right]^{2}} \\
\pi\left(\beta_{B}, \beta_{B}\right)=\frac{a^{2} \beta_{B}\left(2-c \beta_{B}\right)}{2\left[1+\beta_{B}(1+\sigma)\right]^{2}} \\
\pi\left(\beta_{A}, \beta_{B}\right)=\frac{a^{2} \beta_{A}\left(2-c \beta_{A}\right)\left[1+\beta_{B}(1-\sigma)\right]^{2}}{2\left[1+\beta_{B}+\beta_{A}\left(1+\beta_{B}\left(1-\sigma^{2}\right)\right)\right]^{2}} \\
\pi\left(\beta_{B}, \beta_{A}\right)=\frac{a^{2} \beta_{B}\left(2-c \beta_{B}\right)\left[1+\beta_{A}(1-\sigma)\right]^{2}}{2\left[1+\beta_{B}+\beta_{A}\left(1+\beta_{B}\left(1-\sigma^{2}\right)\right)\right]^{2}}
\end{gathered}
$$

so that (31) becomes:

$$
\pi\left(\beta_{A}, \beta_{A}\right)-\pi\left(\beta_{B}, \beta_{A}\right) \geq(\leq) \pi\left(\beta_{A}, \beta_{B}\right)-\pi\left(\beta_{B}, \beta_{B}\right)
$$

Now treating (36) as a strict equality and solving it for $\beta_{A}$, we have that the resulting equation

$$
\pi\left(\beta_{A}, \beta_{A}\right)-\pi\left(\beta_{B}, \beta_{A}\right)=\pi\left(\beta_{A}, \beta_{B}\right)-\pi\left(\beta_{B}, \beta_{B}\right)
$$

can be rewritten as

$$
\Phi\left(\beta_{B}, c, \sigma\right) \beta_{A}^{2}+\Omega\left(\beta_{B}, c, \sigma\right) \beta_{A}+\Lambda\left(\beta_{B}, c, \sigma\right)=0
$$

where $\Phi(\cdot), \Omega(\cdot)$ and $\Lambda(\cdot)$ are polynomials. Moreover,

$$
\begin{gathered}
\Phi\left(\beta_{B}, c, \sigma\right) \equiv c(2+\sigma)+2 \beta_{B}(1+\sigma)[2+3 c+\sigma(1-\sigma)] \\
+2 \beta_{B}^{2}(1+\sigma)^{2}\left[2\left(1-\sigma^{2}\right)+c(2-\sigma)\right]>0 .
\end{gathered}
$$

Equation (38) is solved by $\beta_{A}=\beta_{B}$ and

$$
\beta_{A}^{ \pm}=\frac{-\beta_{B}\left[2 c(2+\sigma)+\beta_{B} \gamma\right]+2+\sigma-c \pm\left[1+\beta_{B}(1+\sigma)\right] \sqrt{\eta}}{2 \beta_{B}(1+\sigma)(\phi+3 c)+c(2+\sigma)}
$$


where

$$
\begin{gathered}
\gamma \equiv(1+\sigma)[2+3 c+\sigma(1-\sigma)] \\
\eta \equiv(2+c+\sigma)^{2}+2 \beta_{B}\left[4(1+\sigma)+c^{2}-5 \sigma^{2}(1+\sigma)+c\left(2(2+\sigma)-3 \sigma^{2}\right)\right] \\
+\beta_{B}^{2}\left[(2+\sigma(1-\sigma))^{2}+c^{2}\left(1+2 \sigma^{2}\right)+2 c\left(2\left(1+\sigma^{3}\right)+\sigma(1+\sigma)\right)\right] \\
\phi \equiv(1+\sigma)\left[2-\sigma+\beta_{B}\left(2\left(1-\sigma^{2}\right)+c(2-s)\right)\right]
\end{gathered}
$$

It can be easily established that the expressions of $\beta_{A}$ in (40) have the following properties:

$$
\left.\beta_{A}^{+}\right|_{\beta_{B}=0}>0>\left.\beta_{A}^{-}\right|_{\beta_{B}=0}
$$

and

$$
\frac{\partial \beta_{A}^{+}}{\partial \beta_{B}}, \frac{\partial \beta_{A}^{-}}{\partial \beta_{B}}<0
$$

everywhere. Additionally, $\beta_{A}^{+} \gtreqless \beta_{B}$ for all

$$
\beta_{B} \lesseqgtr \frac{\sqrt{4(1+c)+c^{2}-3 \sigma^{2}}-c-\sigma}{c(2-\sigma)+2\left(1-\sigma^{2}\right)}>0
$$

for all $c>0$ and all $\sigma \in(0,1]$.

Since we have to confine to $\beta_{A} \geq \beta_{B}$ and, from (39), we know that $\Phi(\cdot)>0$, the following holds:

Proposition 3 For all $\beta_{A} \geq \max \left\{\beta_{A}^{+}, \beta_{B}\right\}$,

$$
\pi\left(\beta_{A}, \beta_{A}\right)-\pi\left(\beta_{B}, \beta_{A}\right) \geq \pi\left(\beta_{A}, \beta_{B}\right)-\pi\left(\beta_{B}, \beta_{B}\right)
$$

For all $\beta_{A} \in\left[\beta_{B}, \beta_{A}^{+}\right]$,

$$
\pi\left(\beta_{A}, \beta_{A}\right)-\pi\left(\beta_{B}, \beta_{A}\right) \leq \pi\left(\beta_{A}, \beta_{B}\right)-\pi\left(\beta_{B}, \beta_{B}\right)
$$

Therefore, since the sign of (36) is not univocally determined over the entire admissible strategy space, we may conclude that the game in supply functions is neither supermodular nor submodular. Proposition 3 is illustrated in Figure 3, where we must confine to the sub-space $\beta_{A}>\beta_{B}$, in 
which the intersection between the 45 -degree line and $\beta_{A}^{+}$identifies two regions. In region $I, \pi\left(\beta_{A}, \beta_{A}\right)-\pi\left(\beta_{B}, \beta_{A}\right) \leq \pi\left(\beta_{A}, \beta_{B}\right)-\pi\left(\beta_{B}, \beta_{B}\right)$; in region $I I, \pi\left(\beta_{A}, \beta_{A}\right)-\pi\left(\beta_{B}, \beta_{A}\right) \geq \pi\left(\beta_{A}, \beta_{B}\right)-\pi\left(\beta_{B}, \beta_{B}\right)$.

Figure 3 Inequality $\pi\left(\beta_{A}, \beta_{A}\right)-\pi\left(\beta_{B}, \beta_{A}\right) \geq(\leq) \pi\left(\beta_{A}, \beta_{B}\right)-\pi\left(\beta_{B}, \beta_{B}\right)$ in the space $\left(\beta_{B}, \beta_{A}\right)$

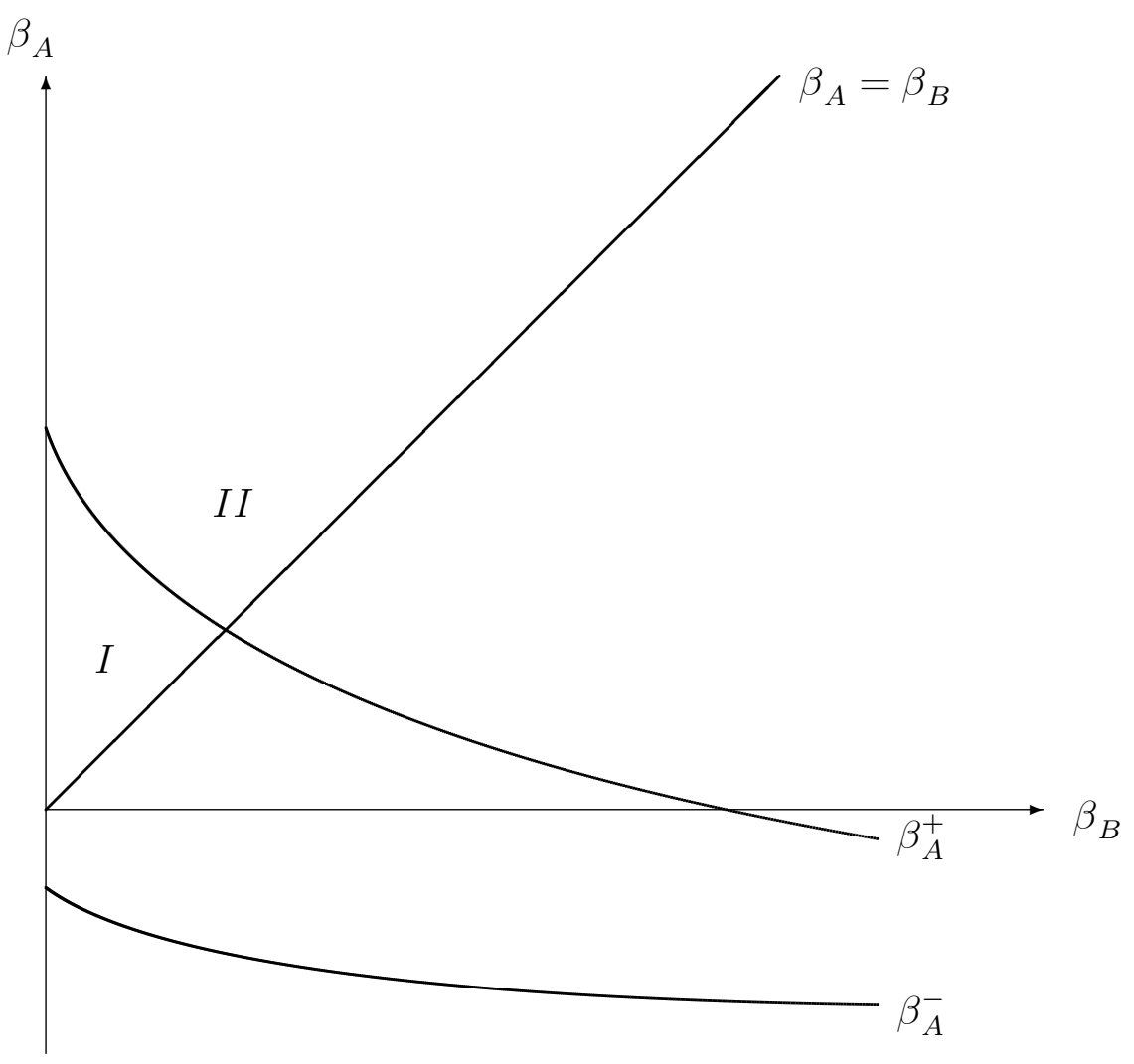

\section{Concluding remarks}

In quantity- and price-setting oligopolies, strategic variables are either strategic substitutes or complements (Bulow et al., 1985) and the games are either 
submodular or supermodular, respectively (e.g., Vives, 1999). We have addressed the same issue under supply function competition (Klemperer and Meyer, 1989). The foregoing analysis has shown that supply functions can labelled as neither strategic complements nor substitutes, because, notwithstanding that the slope of the best reply is always positive, the sign of the cross derivative of marginal profit changes. This conclusion has been confirmed using the lattice-theoretical approach, from which there emerges that the game in the space of supply functions is neither supermodular nor submodular.

\section{Appendix}

The expression (25) can be rewritten as

$$
\begin{gathered}
\Psi=8\left(1+\beta_{j}\right)\left[2(1-\sigma)\left(1+\left(1-\sigma^{2}\right) \beta_{j}\right)+c\left(2-3 \sigma+(2-\sigma)(1-\sigma) \beta_{j}\right)\right] \\
+4\left[c\left(1+\beta_{j}\right)+2 \sigma\left(1+\beta_{j}(1-\sigma)\right)\right]^{2}
\end{gathered}
$$

Therefore, if

$8\left(1+\beta_{j}\right)\left[2(1-\sigma)\left(1+\left(1-\sigma^{2}\right) \beta_{j}\right)+c\left(2-3 \sigma+(2-\sigma)(1-\sigma) \beta_{j}\right)\right] \geq 0$

then (a1) is positive, so that $\operatorname{roots} \beta_{A}^{ \pm} \in \mathbb{R}$ surely. Otherwise, if (a2) is negative, we may again rewrite (a1) to obtain

$$
\begin{gathered}
\Psi=4 \beta_{j}^{2}\left[c^{2}+2 c(2-\sigma(1+\sigma))+4(1-\sigma)^{2}(1+\sigma(1+\sigma))\right] \\
+8 \beta_{j}\left[c^{2}+2(1-\sigma)\left(2+\sigma^{2}\right)+c(4-\sigma(2+\sigma))\right] \\
+4\left[(2+c)(c+2(1-\sigma))+4 \sigma^{2}\right]
\end{gathered}
$$

Solving $\Psi=0$ w.r.t. $\beta_{j}$, we obtain

$$
\beta_{j}^{ \pm}=\frac{f(c, \sigma) \pm \sigma^{2}[c+2(1-\sigma)] \sqrt{3 \mathrm{i}}}{g(c, \sigma)}
$$


where $f(c, \sigma)$ and $g(c, \sigma)$ are polynomials in $c$ and $\sigma$ and i is the imaginary unit. Consequently, equation $\Psi=0$ has no real roots and $\Psi$ has the same sign of the coefficient of $\beta_{j}^{2}$ in (a3):

$$
c^{2}+2 c(2-\sigma(1+\sigma))+4(1-\sigma)^{2}(1+\sigma(1+\sigma))
$$

which is positive for all $c>0$ and $\sigma \in(0,1]$. This proves that, indeed, $\beta_{i+} \neq \beta_{i-}$ and $\beta_{i+}, \beta_{i-} \in \mathbb{R}$. 


\section{References}

[1] Amir, R. (1996), "Cournot Oligopoly and the Theory of Supermodular Games", Games and Economic Behavior, 15, 132-48.

[2] Bolle, F. (1992), "Supply Function Equilibria and the Danger of Tacit Collusion", Energy Economics, 14, 94-102.

[3] Bulow, J.I., J.D. Geanakoplos and P.D. Klemperer (1985), "Multimarket Oligopoly: Strategic Substitutes and Complements", Journal of Political Economy, 93, 488-511.

[4] Ciarreta, A. and C. Gutierrez-Hita (2006), "Supply Function vs Quantity Competition in Supergames", International Journal of Industrial Organization, 24, 773-83.

[5] Delbono, F. and L. Lambertini (2015a), "Ranking Bertrand, Cournot and Supply Function Equilibria in Oligopoly", wp no. 1000, Department of Economics, University of Bologna.

[6] Delbono, F. and L. Lambertini (2015b), "Cournot Retrouvé under Price or Supply Function Competition", wp no. 1003, Department of Economics, University of Bologna.

[7] Delgado, J. and D. Moreno, (2004), "Coalition-Proof Supply Function Equilibria in Oligopoly", Journal of Economic Theory, 114, 231-54.

[8] Green, R.J. and D.M. Newbery (1992), "Competition in the British Electricity Spot Market", Journal of Political Economy, 100, 929-53.

[9] Grossman, S. (1981), "Nash Equilibrium and the Industrial Organization of Markets with Large Fixed Costs", Econometrica, 49, 1149-72.

[10] Holmberg, P., D.M. Newbery and D. Ralph, (2013), "Supply Function Equilibria: Step Functions and Continuous Representations", Journal of Economic Theory, 148, 1509-51. 
[11] Klemperer, P.D. and M.A. Meyer (1989), "Supply Function Equilibria in Oligopoly under Uncertainty", Econometrica, 57, 1243-77.

[12] Milgrom, P. and J. Roberts (1990), "Rationalizability, Learning and Equilibrium in Games with Strategic Complementarities", Econometrica, 58, 1255-77.

[13] Milgrom, P. and C. Shannon (1994), "Monotone Comparative Statics", Econometrica, 62, 157-80.

[14] Singh, N. and X. Vives (1984), "Price and Quantity Competition in a Differentiated Duopoly", RAND Journal of Economics, 15, 546-54.

[15] Tarski, A. (1955), "A Lattice-Theoretical Fixpoint Theorem and its Applications", Pacific Journal of Mathematics, 5, 285-309.

[16] Tirole, J. (1988), The Theory of Industrial Organization, Cambridge, MA, MIT Press.

[17] Topkis, D. (1978), "Minimizing a Submodular Function on a Lattice", Operations Research, 26, 305-21.

[18] Topkis, D. (1979), "Equilibrium Points in Nonzero-Sum n-Person Submodular Games", SIAM Journal on Control and Optimization, 17, 77387.

[19] Vives, X. (1990), "Nash Equilibrium with Strategic Complementarities", Journal of Mathematical Economics, 19, 305-21.

[20] Vives, X. (1999), Oligopoly Pricing. Old Ideas and New Tools, Cambridge, MA, MIT Press.

[21] Vives, X. (2011), "Strategic Supply Function Competition with Private Information", Econometrica, 79, 1919-66. 


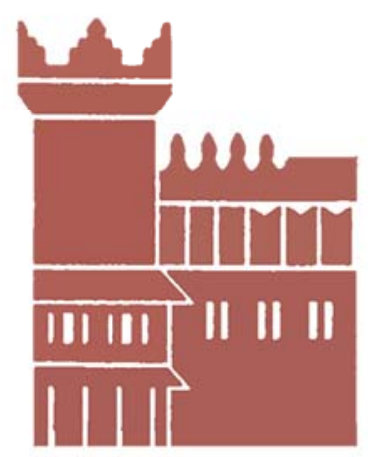

Alma Mater Studiorum - Università di Bologna DEPARTMENT OF ECONOMICS

Strada Maggiore 45

40125 Bologna - Italy

Tel. +39051 2092604

Fax +390512092664

http://www.dse.unibo.it 